\title{
Holographic complexity in charged supersymmetric black holes
}

\author{
Jie Jiang ${ }^{1, \dagger}$ and Ming Zhang $\odot^{2, *}$ \\ ${ }^{1}$ Department of Physics, Beijing Normal University, Beijing 100875, China \\ ${ }^{2}$ Department of Physics, Jiangxi Normal University, Nanchang 330022, China
}

(Received 2 August 2020; accepted 12 September 2020; published 5 October 2020)

\begin{abstract}
For an ordinary charged system, it has been shown that by using the "complexity equals action" (CA) conjecture, the late-time growth rate of the holographic complexity is given by a difference between the value of $\Phi_{H} Q+\Omega_{H} J$ on the inner and outer horizons. In this paper, we study the influence of the chiral anomaly on the complexity of the boundary quantum system. To be specific, we evaluate the complexity equals action holographic complexity of the charged supersymmetric black holes whose bulk action is modified by an additional Chern-Simons term of the electromagnetic fields. As a result, the late-time growth rate of the complexity will be corrected by some additional terms on the inner and outer horizons rather than the ordinary charged black holes. Our work implies that the late-time growth rate of the complexity can carry the information of the chiral anomaly for the boundary system.
\end{abstract}

DOI: 10.1103/PhysRevD.102.084010

\section{INTRODUCTION}

In recent years, there has been a growing interest in the idea that applies the quantum information theory into the gravitational theory in the context of the AdS/CFT correspondence. One of the most famous topics in this direction is the holographic entanglement entropy. However, it has been claimed that the holographic entanglement entropy is not enough to describe the degrees of freedom inside the horizon of the black hole, where the geometry becomes nonstationary. As a result, the quantum circuit complexity of the boundary quantum system, which is defined by the minimal number of elementary gates required to construct a target state for a referent state, has been proposed to describe the information inside the black hole horizon [1,2]. From the holographic viewpoint, Brown et al. suggested that the circuit complexity of the boundary quantum system is given by some classical quantities in the bulk gravitational system, which is called "holographic complexity." Then, there are two main conjectures which are proposed to construct this duality. The first one is the "complexity equals volume" (CV) conjecture [2,3] and the second one is the "complexity equals action" (CA) conjecture [4,5]. These conjectures have attracted a

*Corresponding author. mingzhang@jxnu.edu.cn

jiejiang@mail.bnu.edu.cn

Published by the American Physical Society under the terms of the Creative Commons Attribution 4.0 International license. Further distribution of this work must maintain attribution to the author(s) and the published article's title, journal citation, and DOI. Funded by SCOAP ${ }^{3}$. large number of researchers to study both holographic complexity and circuit complexity in quantum field theory [6-68].

In the present work, we only consider the CA conjecture, which claims that the circuit complexity of the quantum state $\left|\psi\left(t_{L}, t_{R}\right)\right\rangle$ in the boundary system is given by the onshell bulk gravitational action $I_{\mathrm{WDW}}$ within the WheelerDeWitt (WDW) patch, which is enclosed by the past and future light sheets sent into the bulk spacetime from the time slices $t_{L}$ and $t_{R}$, i.e., we have

$$
C_{A}\left(\left|\psi\left(t_{L}, t_{R}\right)\right\rangle\right) \equiv \frac{I_{\mathrm{WDW}}}{\pi \hbar} .
$$

As argued in Ref. [4], there is a bound of the complexity growth rate at the late times

$$
\frac{d C}{d t} \leq \frac{2 M}{\pi \hbar},
$$

which is thought as the Lloyd's bound of the boundary quantum system from the viewpoint of the AdS/CFT correspondence [69].

For the rotating and charged black holes with multiple horizons, a series of works [7-13] has shown that the latetime CA complexity growth rate can be expressed as

$$
\begin{aligned}
\lim _{t \rightarrow \infty} \frac{d C_{A}}{d t}= & \frac{1}{\pi \hbar}\left[\left(M-\Phi_{H}^{(+)} Q-\Omega_{H}^{(+)} J\right)\right. \\
& \left.-\left(M-\Phi_{H}^{(-)} Q-\Omega_{H}^{(-)} J\right)\right],
\end{aligned}
$$

where $Q$ and $J$ are the electric charge and angular momentum of the black holes, $\Omega_{H}^{( \pm)}$and $\Phi_{H}^{( \pm)}$are the 
angular velocity associated with the inner and outer horizons, respectively, and the index $\{( \pm)\}$ presents the quantities evaluated at the "outer" or "inner" horizon. However, all of the above results are obtained in the ordinary charged boundary system. How will things look when we consider an anomalous quantum system? How will be know whether the complexity can carry the information of the anomaly for the boundary system? In this paper, we would like to consider the influence of the chiral anomaly on the complexity. In order to make the boundary system chiral anomaly, it is convenient to introduce an additional Chern-Simons term of the electromagnetic field to the bulk action from the perspective of the AdS/CFT correspondence [70]. Then, based on the inflow mechanism, the gauge invariance in the bulk gravitational theory will cause the boundary quantum system chiral anomaly [70]. Therefore, to study the complexity of the boundary chiral system from the viewpoint of holography, we need a black hole solution in the Einstein-MaxwellChern-Simons theory. For simplicity, in this paper, we only consider the minimal gauged five-dimensional supergravity, which is a special case of the Einstein-Maxwell-ChernSimons theory. The charged rotating black hole solutions in this theory were obtained in [71-74].

The remaining of this paper is organized as follows. In Sec. II, we first review the geometry in the charged supersymmetric black holes and enumerate some basic thermodynamical quantities. In Sec. III, we evaluate the time dependence and the late-time result of the complexity growth rate using the $\mathrm{CA}$ conjecture. Finally, the conclusion and discussion are presented in Sec. IV.

\section{GEOMETRY OF THE FIVE-DIMENSIONAL CHARGED SUPERSYMMETRIC BLACK HOLES}

In this paper, we focus on the five-dimensional minimal gauge supergravity with the bulk action

$$
\begin{aligned}
I_{\mathrm{bulk}}= & \int_{M} \boldsymbol{\epsilon}\left(R+\frac{12}{L^{2}}-\frac{1}{4} \mathcal{F}\right) \\
& +\frac{1}{3 \sqrt{3}} \int_{M} \boldsymbol{F} \wedge \boldsymbol{F} \wedge \boldsymbol{A},
\end{aligned}
$$

where $\boldsymbol{F}=d \boldsymbol{A}$ is the electromagnetic strength tensor, $R$ is the Ricci Scalar, $\boldsymbol{\epsilon}$ is the volume element of the metric $g_{a b}$, $L$ is the cosmological radius, and we denote $\mathcal{F}=F_{a b} F^{a b}$. Varying the action, the equations of motion read

$$
\begin{aligned}
R_{a b}-\frac{1}{2} R g_{a b}-\frac{6}{L^{2}} g_{a b} & =\frac{1}{2}\left(F_{a}{ }^{c} F_{b c}-\frac{g_{a b}}{4} \mathcal{F}\right), \\
\nabla_{a} F^{a b} & =\frac{1}{4 \sqrt{3}} \epsilon^{\mathrm{bcdef}} F_{c d} F_{e f} .
\end{aligned}
$$

Generally, the black hole solutions in this gravitational theory carry two angular momenta. For simplicity, in this following, we only focus on the special case which has two equal angular momenta. The solution is given by [72]

$$
\begin{aligned}
d s^{2} & =-\frac{f}{h} d t^{2}+\frac{d r^{2}}{f}+\frac{r^{2}}{4}\left(\sigma_{1}^{2}+\sigma_{2}^{2}\right)+\frac{r^{2} h}{4}\left(\sigma_{3}-W d t\right)^{2}, \\
\boldsymbol{A} & =-\frac{\sqrt{3} q}{r^{2}}\left(d t-\frac{j}{2} \sigma_{3}\right),
\end{aligned}
$$

in which $\sigma_{1}, \sigma_{2}, \sigma_{3}$ are the usual left-invariant one-fore of the three-sphere and they are given by

$$
\begin{aligned}
& \sigma_{1}=-\sin \psi d \theta+\cos \psi \sin \theta d \phi, \\
& \sigma_{2}=\cos \psi d \theta+\sin \psi \sin \theta d \phi, \\
& \sigma_{3}=d \psi+\cos \theta d \phi,
\end{aligned}
$$

and

$$
\begin{aligned}
& f=1+\frac{r^{2}}{L^{2}}-\frac{2 m(1-\eta)}{r^{2}}+\frac{q^{2}}{r^{4}}\left(1-\frac{j^{2}}{L^{2}}+\frac{2 L^{2} m \chi}{q^{2}}\right), \\
& W=\frac{2 j\left[(2 m-q) r^{2}-q^{2}\right]}{r^{6} h}, \\
& h=1-\frac{j^{2} q^{2}}{r^{6}}+\frac{2 j^{2}(m-q)}{r^{4}}
\end{aligned}
$$

with the quantity

$$
\eta=\frac{j^{2}(m-q)}{m L^{2}} .
$$

The electric charge and angular momenta of the black hole are defined by

$Q=\int_{S_{\infty}}\left(\star \boldsymbol{F}-\frac{1}{\sqrt{3}} \boldsymbol{F} \wedge \boldsymbol{A}\right), \quad J=\int_{S_{\infty}} \star d \varphi$,

where

$$
\varphi^{a}=\left(\frac{\partial}{\partial \psi}\right)^{a}
$$

is the axial Killing vector field and $S_{\infty}$ is a three-sphere at asymptotic infinity. For the charged supersymmetric black hole solution in Eq. (6), the energy, electric charge, and angular momentum are given by

$$
\begin{aligned}
M & =\frac{3 \Omega_{3} m}{4}\left(1+\frac{\eta}{3}\right), \\
Q & =\frac{\sqrt{3} \Omega_{3} q}{4}, \\
J & =\frac{\Omega_{3} j(2 m-q)}{4},
\end{aligned}
$$

in which $\Omega_{3}=16 \pi^{2}$ is the volume of the unit three-sphere with the line element 


$$
d \Omega_{3}^{2}=d \theta^{2}+\sin ^{2} \theta d \phi^{2}+\cos ^{2} \theta d \psi^{2} .
$$

As mentioned in the Introduction, in the present work, we only consider the spacetime solution which describes a black hole with two Killing horizons. The inner and outer horizon is determined by $f\left(r_{ \pm}\right)=0$. From the line element of this solution, we can see that if there are some regions outside the horizon such that $h(r) \leq 0$, there will exist some naked closed timelike curves lying outside the horizon. Then, the causality of this spacetime will be destroyed. Moreover, if $h(r)<0$ between the inner and outer horizon, the sign of the metric will become $(-,-,-,+,+)$, which is unphysical and cannot be used to describe a spacetime geometry. Taking these into account, in the following, we only focus on the black hole geometry where $h(r)>0$ outside the inner horizon. In this case, the Killing vector fields which generates the inner and outer horizon are given by

$$
k_{( \pm)}^{a}=\left(\frac{\partial}{\partial t}\right)^{a}+\Omega_{H}^{( \pm)}\left(\frac{\partial}{\partial \psi}\right)^{a}
$$

in which $\Omega_{H}^{( \pm)}=W\left(r_{ \pm}\right)$presents the angular velocities of the inner and outer horizons. The corresponding temperature, entropy, and electric potential are given by

$$
\begin{aligned}
S^{( \pm)} & =\frac{\Omega_{3} \pi r_{ \pm}^{3} \sqrt{h\left(r_{ \pm}\right)}}{2}, \\
T^{( \pm)} & =\frac{f^{\prime}\left(r_{ \pm}\right)}{4 \pi \sqrt{h\left(r_{ \pm}\right)}}, \quad \Phi_{H}^{( \pm)}=\frac{\sqrt{3} q\left(2-a \Omega_{H}^{( \pm)}\right)}{2 r_{ \pm}^{2}} .
\end{aligned}
$$

\section{COMPLEXITY GROWTH RATE IN CA CONJECTURE}

In this section, we would like to evaluate the growth rate of the holographic complexity for the above charged supersymmetric black holes based on the CA conjecture. That is to say, we need to evaluate the on-shell action within the WDW patch. As suggested by Lehner et al. in [6], the total on-shell action should include not only the bulk action but the surface terms, joint terms, and counterterms as well. Here the surface terms and joint terms are introduced to make the variational principle well posed when the bulk region has a nonsmooth boundary. The counterterms are added to make the total action invariant under the reparametrization of the null generator on the null segment. According to [6], the total action can be expressed as

$$
\begin{aligned}
I= & I_{\text {bulk }}+2 \int_{B} d^{4} x \sqrt{|h|} K \pm 2 \int_{S} d^{3} \theta \sqrt{\sigma} \eta \\
& +2 \int_{N} d \lambda d^{3} \theta \sqrt{\gamma} \kappa+2 \int_{N} d \lambda d^{3} \theta \sqrt{\gamma} \Theta \ln \left(\ell_{\mathrm{ct}} \Theta\right),
\end{aligned}
$$

where $B$ and $N$ are the non-null and null segments of the boundary $\partial M$ separately, and $S$ is a three-dimensional joint of the nonsmooth boundary. Here, $h_{a b}$ and $K$ are the induced metric and the trace of the extrinsic curvature on the non-null surface $B, \eta$ is a transformation parameter on the joint $S, \gamma_{a b}$ is the induced metric on the cross section of the null segment $N, \lambda$ is the parameter of the null generator $k^{a}$ on the null segment, the parameter $\kappa$ is given by $k^{a} \nabla_{a} k^{b}=\kappa k^{b}$ and it measures the failure of $\lambda$ to be an affine parameter, $\Theta=\nabla_{a} k^{a}$ is the expansion scalar of the null generator, and $\ell_{\mathrm{ct}}$ is some arbitrary constant parameter.

In Fig. 1, we show the change of the WDW patch in the charged supersymmetric black holes which has two Killing horizons. Considering that the spacetime is invariant under the shift transformation $t_{R} \rightarrow t_{R}-\delta t, t_{L} \rightarrow t_{L}+\delta t$, without loss of generality, we fix the left boundary time $t_{L}=0$ and only vary the right boundary time $t_{R}=t$ of the WDW patch. Since we only focus on the growth rate of the action within the WDW patch, we can neglect the higher-order term $\mathcal{O}(\delta t)$ of $\delta t$. From Fig. 1, we can see that the bulk region $\delta M_{m}^{( \pm)}$can be generated by the horizon Killing vector $k_{( \pm)}^{a}$ through the null segment $N_{m}^{( \pm)}$under the firstorder approximation of $\delta t$ (Generally, it can be generated by the Killing vector $k_{( \pm)}^{a}+C \varphi^{a}$ with any constant $C$ because the null segment $N_{m}^{( \pm)}$is invariant under the axial transformation). Moreover, we shall choose $\lambda$ to be the affine parameter of the null generators. As a result, the surface term vanishes on all null segments. With these in mind, the change of the total action is given by

$$
\delta I_{\mathrm{WDW}}=I_{\delta M_{m}^{(-)}}-I_{\delta M_{m}^{(+)}}+\delta I_{S_{m}^{(-)}}-\delta I_{S_{m}^{(+)}}+\delta I_{\mathrm{ct}} .
$$

\section{A. Bulk contributions}

We start by evaluating the contributions from the bulk action in the region $\delta M_{m}^{( \pm)}$. Because the calculations are same for $\delta M_{m}^{(-)}$and $\delta M_{m}^{(+)}$, we will neglect the index $( \pm)$ for all the quantities. From the equations of motion, we can further obtain

$$
\begin{aligned}
R & =\frac{1}{12} \mathcal{F}-\frac{20}{L^{2}}, \\
R_{a b} & =\frac{1}{2} F_{a}{ }^{c} F_{b c}-g_{a b}\left(\frac{\mathcal{F}}{12}+\frac{4}{L^{2}}\right) .
\end{aligned}
$$

Then, the bulk contribution from the region $\delta M_{m}^{( \pm)}$gives

$$
\begin{aligned}
I_{\delta M_{m}} & =-\left(\frac{8}{L^{2}}+\frac{\mathcal{F}}{6}\right) \int_{\delta M} \sqrt{-g} d^{5} x+\frac{1}{3 \sqrt{3}} \int_{\delta M} \boldsymbol{F} \wedge \boldsymbol{F} \wedge \boldsymbol{A} \\
& =-\delta t\left(\frac{8}{L^{2}}+\frac{\mathcal{F}}{6}\right) \int_{N} \star \xi+\frac{\delta t}{3 \sqrt{3}} \int_{N} \xi \cdot(\boldsymbol{F} \wedge \boldsymbol{F} \wedge \boldsymbol{A}) .
\end{aligned}
$$

Considering the facts that $k^{a}$ is a Killing vector and the sum of cyclic permutations of the last three indices in $R^{a} b c d$ vanishes, we have the following identity: 


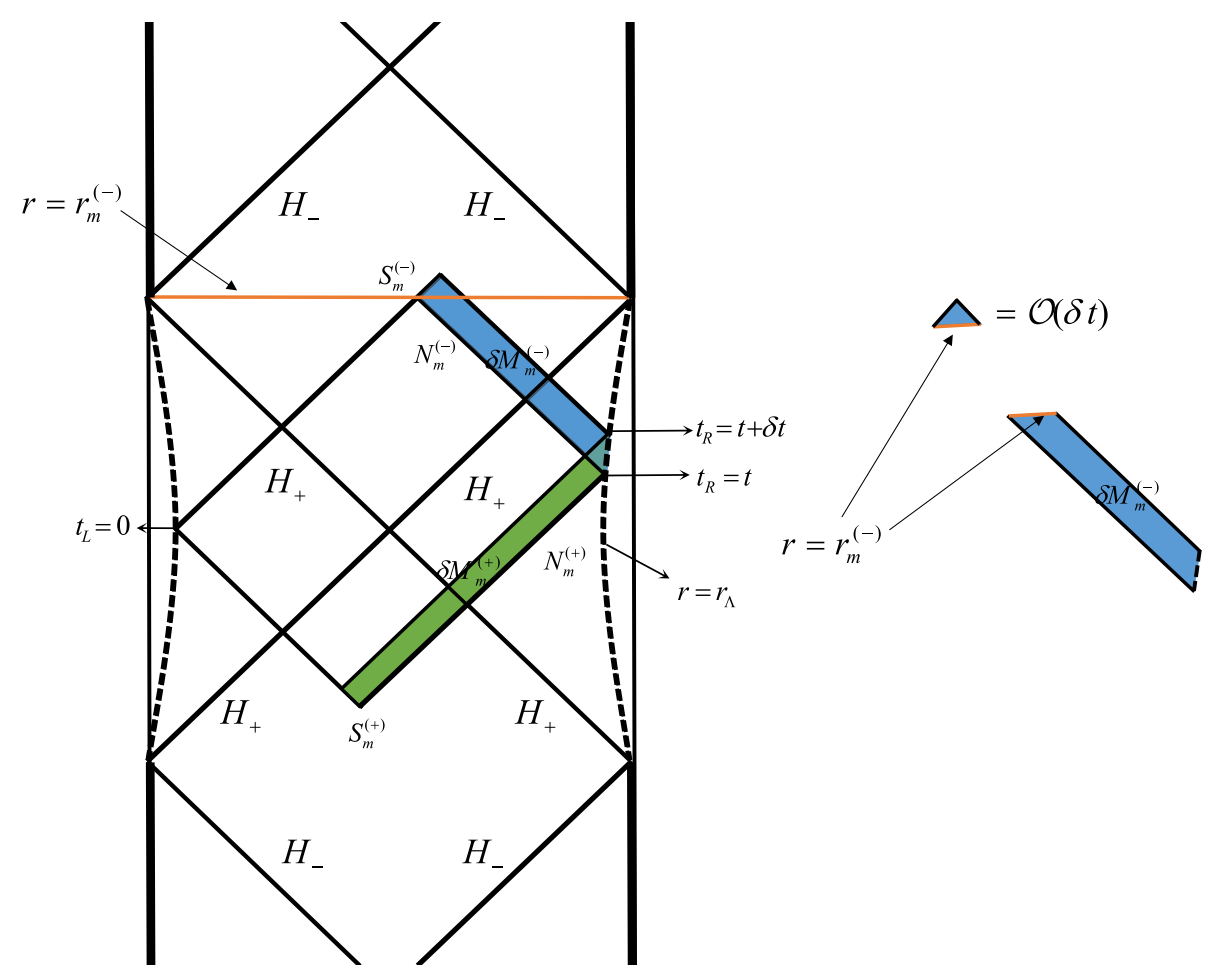

FIG. 1. A spacetime diagram of charged supersymmetric black holes with two Killing horizons. In the right panel, we show the change of the Wheeler-DeWitt patch in this spacetime in which we fix the left boundary time $t_{L}=0$ and vary the right boundary time $t_{R}=t$. The dashed lines denote the cutoff surface $r=r_{\Lambda}$ at asymptotic infinity to regulate the divergence near the anti-de Sitter (AdS) boundary. In the right panel, we show that the bulk region $\delta M_{m}^{(-)}$of the WDW patch can be generated by the Killing vector $k_{(-)}^{a}$ through $N_{m}^{(-)}$under the first-order approximation of $\delta t$.

$$
\begin{aligned}
\nabla_{a} \nabla^{a} k^{b} & =-R^{b}{ }_{a} k^{a} \\
& =\left(\frac{\mathcal{F}}{12}+\frac{4}{L^{2}}\right) k^{b}-\frac{1}{2} k^{a} F_{a c} F^{b c}
\end{aligned}
$$

For the second term, we have

$$
\begin{aligned}
k^{a} F_{a c} F^{b c} & =k^{a}(d \boldsymbol{A})_{a c} F^{b c} \\
& =\mathcal{L}_{k} \boldsymbol{A}_{c} F^{b c}+\nabla_{c} \Phi F^{b c} \\
& =-\nabla_{a}\left(\Phi F^{a b}\right)+\Phi \nabla_{a} F^{a b} \\
& =-\nabla_{a}\left(\Phi F^{a b}\right)+\frac{\Phi}{4 \sqrt{3}} \epsilon^{\mathrm{bcdef}} F_{c d} F_{e d},
\end{aligned}
$$

where we have denoted

$$
\Phi^{( \pm)}=-A_{a} k_{ \pm}^{a} .
$$

Then, we can get

$2 \nabla_{a} \nabla^{a} k^{b}=\left(\frac{\mathcal{F}}{6}+\frac{8}{L^{2}}\right) k^{b}+\nabla_{a}\left(\Phi F^{a b}\right)-\frac{\Phi}{4 \sqrt{3}} \epsilon^{\text {bcdef }} F_{c d} F_{e d}$.

Using the language of differential forms, the above identity can be expressed as

$$
-\left(\frac{\mathcal{F}}{6}+\frac{8}{L^{2}}\right) \star \boldsymbol{k}=d(\star d \boldsymbol{k}-\Phi \star \boldsymbol{F})+\frac{\Phi}{\sqrt{3}} \boldsymbol{F} \wedge \boldsymbol{F} .
$$

On the other hand, we have

$$
\begin{aligned}
k \cdot(\boldsymbol{F} \wedge \boldsymbol{F} \wedge \boldsymbol{A}) & =2(k \cdot \boldsymbol{F}) \wedge \boldsymbol{F} \wedge \boldsymbol{A}-\Phi \boldsymbol{F} \wedge \boldsymbol{F} \\
& =2 d \Phi \wedge \boldsymbol{F} \wedge \boldsymbol{A}-\Phi \boldsymbol{F} \wedge \boldsymbol{F} \\
& =2 d(\Phi \boldsymbol{F} \wedge \boldsymbol{A})-3 \Phi \boldsymbol{F} \wedge \boldsymbol{F}
\end{aligned}
$$

Summing the above results, we can further obtain

$$
\begin{aligned}
\frac{I_{\delta M_{m}}}{\delta t} & =\int_{N} d\left(\star d \boldsymbol{k}-\Phi \star \boldsymbol{F}+\frac{2}{3 \sqrt{3}} \Phi \boldsymbol{F} \wedge \boldsymbol{A}\right) \\
& =\int_{\partial N}\left(\star d \boldsymbol{k}-\Phi \star \boldsymbol{F}+\frac{2}{3 \sqrt{3}} \Phi \boldsymbol{F} \wedge \boldsymbol{A}\right) \\
& =\int_{S_{\infty}} \star d \boldsymbol{k}-\int_{S_{m}} \star d \boldsymbol{k}+\Phi\left(r_{m}\right) \int_{S_{m}}\left(\star \boldsymbol{F}-\frac{2}{3 \sqrt{3}} \boldsymbol{F} \wedge \boldsymbol{A}\right) \\
& =\int_{S_{\infty}} \star d \boldsymbol{\xi}+\Omega_{H} J+\Phi\left(r_{m}\right) Q-\chi\left(r_{m}\right) \Phi\left(r_{m}\right)+\mathcal{P}\left(r_{m}\right) .
\end{aligned}
$$

where we have denoted 


$$
\begin{aligned}
\chi(r) & =-\frac{1}{3 \sqrt{3}} \int_{S_{r}} \boldsymbol{F} \wedge \boldsymbol{A}=\frac{\Omega_{3} q^{2} a^{2}}{4 \sqrt{3} r^{4}}, \\
\mathcal{P}^{( \pm)}(r) & =-\int_{S_{r}} \star d \boldsymbol{k}_{ \pm} \\
& =\frac{\Omega_{3} r^{3}}{32 h}\left[4 h f^{\prime}-4 f h^{\prime}+h^{3} r^{2} W^{\prime}\left(\Omega_{H}^{( \pm)}-W\right)\right],
\end{aligned}
$$

in which $S_{r}$ is a three-sphere with radius $r$. After completing all of the indexes, the bulk contributions yield

$$
\begin{aligned}
\frac{d I_{\text {bulk }}}{d t}= & \frac{I_{\delta M_{m}^{(-)}}}{\delta t}-\frac{I_{\delta M_{m}^{(-)}}}{\delta t} \\
= & \left(\Omega_{H}^{(-)}-\Omega_{H}^{(+)}\right) J+\left[\Phi^{(-)}\left(r_{m}^{(-)}\right)-\Phi^{(+)}\left(r_{m}^{(+)}\right)\right] Q \\
& -\left[\chi\left(r_{m}^{(-)}\right) \Phi^{(-)}\left(r_{m}^{(-)}\right)-\chi\left(r_{m}^{(+)}\right) \Phi^{(+)}\left(r_{m}^{(+)}\right)\right] \\
& +\mathcal{P}^{(-)}\left(r_{m}^{(-)}\right)-\mathcal{P}^{(+)}\left(r_{m}^{(+)}\right) .
\end{aligned}
$$

At the late times $t=t_{R} \rightarrow \infty$, and we have $r_{m}^{( \pm)} \rightarrow r_{ \pm}$. Then, the growth rate of the bulk action becomes

$$
\begin{aligned}
\lim _{t \rightarrow \infty} \frac{d I_{\mathrm{bulk}}}{d t}= & \left(\Omega_{H}^{(-)} J+\Phi_{H}^{(-)} Q-\chi_{H}^{(-)} \Phi_{H}^{(-)}+T^{(-)} S^{(-)}\right) \\
& -\left(\Omega_{H}^{(+)} J+\Phi_{H}^{(+)} Q-\chi_{H}^{(+)} \Phi_{H}^{(+)}+T^{(+)} S^{(+)}\right),
\end{aligned}
$$

where we have denoted

$$
\chi_{H}^{( \pm)}=\chi\left(r_{ \pm}\right)
$$

\section{B. Joint contributions}

We next turn to calculate the joint contributions from meeting points $S_{m}^{( \pm)}$. We first focus on the joint $S_{m}^{(+)}$which is formed by the intersection of the past right and past left null segments. From the line element (6), it is not hard to see

$$
\begin{aligned}
& \left(k_{1}\right)_{a}=(d v)_{a}+\frac{1}{\tilde{f}(r)}(d r)_{a}, \\
& \left(k_{2}\right)_{a}=(d v)_{a}-\frac{1}{\tilde{f}(r)}(d r)_{a}
\end{aligned}
$$

with

$$
\tilde{f}(r)=\frac{f(r)}{\sqrt{h(r)}}
$$

are the affinely null generator of the past right and past left null segments separately. The null hypersurfaces in KerrNewman-(A)dS spacetimes have also been studied in $[75,76]$. The transformation parameter of this joint is given by $\eta=\ln \left(\left|k_{1} \cdot k_{2}\right| / 2\right)$ [6]. Using the above null generators, we can further obtain

$$
k_{1} \cdot k_{2}=-\frac{2 h(r)}{f(r)}
$$

Then, the joint contribution from $S_{m}^{(+)}$is given by

$$
I_{S_{m}^{(+)}}=-\left.\frac{\Omega r^{3} \sqrt{h}}{4} \ln [-f / h]\right|_{r=r_{m}^{(+)}} .
$$

From Eq. (31), we can see that these two segments are determined by $t+r^{\star}(r)=t_{R}$ and $t-r^{\star}(r)=t_{L}=0$. Here $r^{\star}(r)$ is the tortoise coordinate and it is defined by

$$
r^{\star}(r)=-\int_{r}^{\infty} \frac{d r}{\tilde{f}(r)},
$$

where this range of integration is chosen to indicate that the coordinate satisfies the boundary condition

$$
\lim _{r \rightarrow \infty} r_{\star}(r)=0 .
$$

According to [11], it can be written as

$$
\begin{aligned}
r^{\star}(r)= & \frac{\ln \left(\left|r-r_{+}\right| / r\right)}{g\left(r_{+}\right)\left(r_{+}-r_{-}\right)}-\frac{\ln \left(\left|r-r_{-}\right| / r\right)}{g\left(r_{-}\right)\left(r_{+}-r_{-}\right)} \\
& -\frac{1}{r_{+}-r_{-}} \int_{r}^{\infty} G(r) d r,
\end{aligned}
$$

with

$$
\begin{aligned}
g(r) & =\frac{\tilde{f}(r)}{\left(r-r_{+}\right)\left(r-r_{-}\right)}, \\
G(r) & =\frac{g\left(r_{+}\right) r-g(r) r_{+}}{g\left(r_{+}\right) g(r) r\left(r-r_{+}\right)}-\frac{g\left(r_{-}\right) r-g(r) r_{-}}{g\left(r_{-}\right) g(r) r\left(r-r_{-}\right)} .
\end{aligned}
$$

From the above discussion, the radius of the meeting point $S_{m}^{(+)}$can be further obtained,

$$
r^{\star}\left(r_{m}^{(+)}\right)=-\frac{t}{2}
$$

Then, the change rate of this dynamical point can be read off

$$
\frac{d r_{m}^{(+)}}{d t}=-\frac{1}{2} \tilde{f}\left(r_{m}^{(+)}\right)
$$

The time derivative of the joint action can be expressed by

$$
\frac{d I_{S_{m}^{(+)}}}{d t}=\frac{\Omega_{3} r^{3}\left(h f^{\prime}-f h^{\prime}\right)}{8 h}+\left.\frac{\Omega_{3} r^{2}\left(6 h+r h^{\prime}\right) f \ln [-f / h]}{16 h}\right|_{r_{m}^{(+)}} .
$$

With a similar calculation, we can also obtain the contribution from the joint $S_{m}^{(-)}$and the growth rate of the joint contribution is given by 

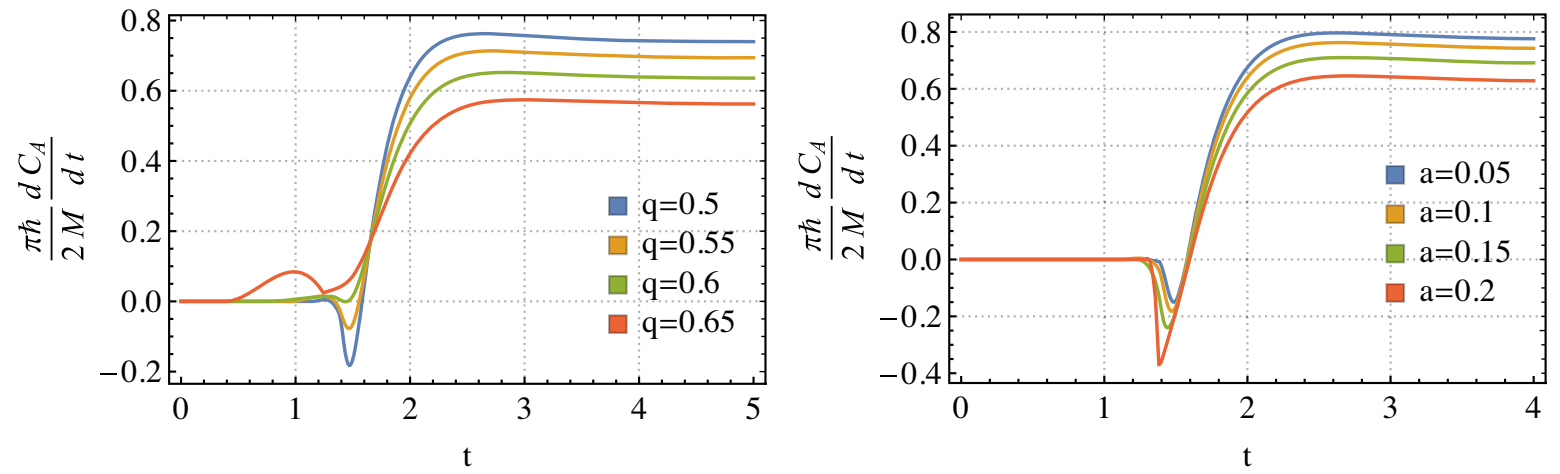

FIG. 2. Plots show the time dependence of the complexity growth rate. In the left panel, we vary the charge parameter $q$ and fix $m=1$, $L=1, l_{\mathrm{ct}}=0.5, a=0.1$. In the right panel, we vary the angular momenta parameter $a$ and fix $m=1, L=1, l_{\mathrm{ct}}=0.5, q=0.5$.

$\frac{d I_{\text {joint }}}{d t}=\frac{\Omega_{3} r^{3}\left(h f^{\prime}-f h^{\prime}\right)}{8 h}+\left.\frac{\Omega_{3} r^{2}\left(6 h+r h^{\prime}\right) f \ln [-f / h]}{16 h}\right|_{r_{m}^{(-)}} ^{r_{m}^{(+)}}$,

where we have used the relation

$$
r^{\star}\left(r_{m}^{(-)}\right)=\frac{t}{2}
$$

At the late times, we have

$$
\lim _{t \rightarrow \infty} \frac{d I_{\text {joint }}}{d t}=T^{(+)} S^{(+)}-T^{(-)} S^{(-)}
$$

We can see that this term will cancel the $T S$ term in the bulk contributions.

\section{Counterterm contributions}

Finally, we are going to evaluate the counterterm contributions. We first consider the past right null segment. From Eq. (31), the expansion of the null generator on this segment gives

$$
\Theta=\frac{6 h+r h^{\prime}}{2 r \sqrt{h}} .
$$

Then, the counterterm of the past right null segment can be shown as

$I_{\mathrm{ct}}^{(\mathrm{pr})}=\left.\frac{\Omega_{3}}{8} \int_{r_{m}^{(+)}(\lambda)}^{r_{\Lambda}(\lambda)} d \lambda r^{2}\left(6 h+r h^{\prime}\right) \ln \left(\frac{\left(6 h+r h^{\prime}\right) \ell_{\mathrm{ct}}}{2 r \sqrt{h}}\right)\right|_{r=r(\lambda)}$.

Here $\lambda$ is the affine parameter of the null generator $k_{1}^{a}=(\partial / \partial \lambda)^{a}$. Together with Eq. (31), we can find that $r^{\prime}(\lambda)=\sqrt{h}$, which implies that $d \lambda=d r / \sqrt{h}$. Then, we have
$I_{\mathrm{ct}}^{(\mathrm{pr})}=\frac{\Omega_{3}}{8} \int_{r_{m}^{(+)}}^{r_{\Lambda}} d r \frac{r^{2}\left(6 h+r h^{\prime}\right)}{\sqrt{h}} \ln \left(\frac{\left(6 h+r h^{\prime}\right) \ell_{\mathrm{ct}}}{2 r \sqrt{h}}\right)$.

Using Eq. (39), we can further obtain

$\frac{d I_{\mathrm{ct}}^{(\mathrm{pr})}}{d t}=\left.\frac{\Omega_{3} r^{2}\left(6 h+r h^{\prime}\right) f}{16 h} \ln \left(\frac{\left(6 h+r h^{\prime}\right) \ell_{\mathrm{ct}}}{2 r \sqrt{h}}\right)\right|_{r_{m}^{(+)}}$.

Again, we can obtain the counterterm contributions from other segments and the final result is given by

$$
\frac{d I_{\mathrm{ct}}}{d t}=\left.\frac{\Omega_{3} r^{2}\left(6 h+r h^{\prime}\right) f}{8 h} \ln \left(\frac{\left(6 h+r h^{\prime}\right) \ell_{\mathrm{ct}}}{2 r \sqrt{h}}\right)\right|_{r_{m}^{(-)}} ^{r_{m}^{(+)}} .
$$

\section{Complexity growth rate}

Summing all the previous results and using the CA conjecture in Eq. (1), one can further obtain

$$
\begin{aligned}
\pi \hbar \frac{d C_{A}}{d t}= & \left(\Omega_{H}^{(-)}-\Omega_{H}^{(+)}\right) J+\left[\Phi^{(-)}\left(r_{m}^{(-)}\right)-\Phi^{(+)}\left(r_{m}^{(+)}\right)\right] Q \\
& -\chi\left(r_{m}^{(-)}\right) \Phi^{(-)}\left(r_{m}^{(-)}\right)+\chi\left(r_{m}^{(+)}\right) \Phi^{(+)}\left(r_{m}^{(+)}\right) \\
& +\tilde{\mathcal{P}}^{(-)}\left(r_{m}^{(-)}\right)-\tilde{\mathcal{P}}^{(+)}\left(r_{m}^{(+)}\right) \\
& -\left[\frac{\Omega_{3} r^{2}\left(6 h+r h^{\prime}\right) f}{16 h} \ln \left(-\frac{\left(6 h+r h^{\prime}\right)^{2} f \ell_{\mathrm{ct}}^{2}}{4 r^{2} h^{2}}\right)\right]_{r_{m}^{(+)}}^{r_{m}^{(-)}}
\end{aligned}
$$

where we have denoted

$$
\tilde{\mathcal{P}}^{( \pm)}(r)=\frac{\Omega_{3} h^{2} r^{5} W^{\prime}}{32}\left(\Omega_{H}^{( \pm)}-W\right) .
$$

In Fig. 2, we show the time dependence of the CA complexity growth rate in the charged supersymmetric black holes. This figure shows a similar behavior with the case of the Reissner-Nordström (RN)-AdS black holes where the late-time value is approached above. 
Finally, we consider the late-time limit of the complexity growth rate. From Eq. (50), it is easy to get

$$
\begin{aligned}
\lim _{t \rightarrow \infty} \frac{d C_{A}}{d t}= & \frac{1}{\pi \hbar}\left[\left(\Omega_{H}^{(-)} J+\Phi_{H}^{(-)} Q-\chi_{H}^{(-)} \Phi_{H}^{(-)}\right)\right. \\
& \left.-\left(\Omega_{H}^{(+)} J+\Phi_{H}^{(+)} Q-\chi_{H}^{(+)} \Phi_{H}^{(+)}\right)\right]
\end{aligned}
$$

The above results show the difference from the ordinary charged system as shown in Eq. (3). Here the late-time growth rate will be corrected by some additional terms which are evaluated on the inner and outer horizons, i.e., $\chi_{H}^{(+)} \Phi_{H}^{(+)}-\chi_{H}^{(-)} \Phi_{H}^{(-)}$.

\section{CONCLUSION AND DISCUSSION}

In this paper, we considered the five-dimensional minimal gauged supergravity, which is a special case of the Einstein-Maxwell-Chern-Simons theory. From the perspective of AdS/CFT, the dual bound system of this bulk gravity is a real anomaly. To study the influence of the chiral anomaly of the boundary system to the complexity, we evaluated the growth rate of the holographic complexity in charged and rotating supersymmetric black holes by using the CA conjecture. As a result, we found that the time dependence of the complexity growth rate shares similar behavior as the cases of the RN black holes. However, the late-time rate is different from the result (3) of the ordinary charged system. Here it is corrected by an additional term $\chi_{H} \Phi_{H}$ which is evaluated on the inner and outer horizons. These imply that the late-time growth rate of the complexity carries some information of the chiral anomaly for the boundary quantum system. Moreover, from the above calculations, it is not hard to verify that the additional corrections $\alpha\left(\chi_{H}^{(+)} \Phi_{H}^{(+)}-\chi_{H}^{(-)} \Phi_{H}^{(-)}\right)$will also appear in a general Einstein-Maxwell-Chern-Simons gravity, which includes a general Chern-Simons term

$$
I_{\mathrm{CS}}=\frac{\alpha}{2 \sqrt{3}} \int_{M} \boldsymbol{F} \wedge \boldsymbol{F} \wedge \boldsymbol{A},
$$

with a coupling constant $\alpha$. Our work strongly implies that the anomaly of the boundary will play an important role in complexity.

\section{ACKNOWLEDGMENTS}

Jie Jiang is supported by the National Natural Science Foundation of China (Grants No. 11775022 and NO. 11873044). Ming Zhang is supported by the Initial Research Foundation of Jiangxi Normal University with Grant No. 12020023.
[1] S. Aaronson, The complexity of quantum states and transformations: From quantum money to black holes, arXiv: 1607.05256.

[2] L. Susskind, Computational complexity and black hole horizons, Fortschr. Phys. 64, 24 (2016).

[3] D. Stanford and L. Susskind, Complexity and shock wave geometries, Phys. Rev. D 90, 126007 (2014).

[4] A. R. Brown, D. A. Roberts, L. Susskind, B. Swingle., and Y. Zhao, Holographic Complexity Equals Bulk Action?, Phys. Rev. Lett. 116, 191301 (2016).

[5] A. R. Brown, D. A. Roberts, L. Susskind, B. Swingle, and Y. Zhao, Complexity action, and black holes, Phys. Rev. D 93, 086006 (2016).

[6] L. Lehner, R. C. Myers, E. Poisson, and R. D. Sorkin, Gravitational action with null boundaries, Phys. Rev. D 94, 084046 (2016).

[7] R. G. Cai, S. M. Ruan, S. J. Wang, R. Q. Yang, and R. H. Peng, Action growth for AdS black holes, J. High Energy Phys. 09 (2016) 161.

[8] W. D. Guo, S. W. Wei, Y. Y. Li, and Y.X. Liu, Complexity growth rates for AdS black holes in massive gravity and $f(R)$ gravity, Eur. Phys. J. C 77, 904 (2017).

[9] P. Wang, H. Yang, and S. Ying, Action growth in $f(R)$ gravity, Phys. Rev. D 96, 046007 (2017).
[10] W. J. Pan and Y.C. Huang, Holographic complexity and action growth in massive gravities, Phys. Rev. D 95, 126013 (2017).

[11] D. Carmi, S. Chapman, H. Marrochio, R. C. Myers, and S. Sugishita, On the time dependence of holographic complexity, J. High Energy Phys. 11 (2017) 188.

[12] Z. Y. Fan and M. Guo, Holographic complexity and thermodynamics of AdS black holes, Phys. Rev. D 100, 026016 (2019).

[13] J. Jiang, Action growth rate for a higher curvature gravitational theory, Phys. Rev. D 98, 086018 (2018).

[14] Z. Y. Fan and M. Guo, On the Noether charge and the gravity duals of quantum complexity, J. High Energy Phys. 08 (2018) 031.

[15] Y.S. An, R. G. Cai, and Y. Peng, Time dependence of holographic complexity in Gauss-Bonnet gravity, Phys. Rev. D 98, 106013 (2018).

[16] Y.S. An and R. H. Peng, Effect of the dilaton on holographic complexity growth, Phys. Rev. D 97, 066022 (2018).

[17] A. Reynolds and S. F. Ross, Complexity in de Sitter space, Classical Quantum Gravity 34, 175013 (2017).

[18] S. Chapman, H. Marrochio, and R. C. Myers, Complexity of formation in holography, J. High Energy Phys. 01 (2017) 062. 
[19] X. H. Feng and H. S. Liu, Holographic complexity growth rate in Horndeski theory, Eur. Phys. J. C 79, 40 (2019).

[20] D. Carmi, R. C. Myers, and P. Rath, Comments on holographic complexity, J. High Energy Phys. 03 (2017) 118.

[21] M. Alishahiha, Holographic complexity, Phys. Rev. D 92, 126009 (2015).

[22] C. A. Agon, M. Headrick, and B. Swingle, Subsystem complexity and holography, J. High Energy Phys. 02 (2019) 145.

[23] O. Ben-Ami and D. Carmi, On volumes of subregions in holography and complexity, J. High Energy Phys. 11 (2016) 129.

[24] J. Jiang and M. Zhang, Holographic complexity of the electromagnetic black hole, Eur. Phys. J. C 80, 85 (2020).

[25] Y. Zhao, Uncomplexity and black hole geometry, Phys. Rev. D 97, 126007 (2018).

[26] Z. Fu, A. Maloney, D. Marolf, H. Maxfield, and Z. Wang, Holographic complexity is nonlocal, J. High Energy Phys. 02 (2018) 072.

[27] M. Alishahiha, A. Faraji Astaneh, M. R. Mohammadi Mozaffar, and A. Mollabashi, Complexity growth with Lifshitz scaling and hyperscaling violation, J. High Energy Phys. 07 (2018) 042.

[28] J. Couch, S. Eccles, W. Fischler, and M. L. Xiao, Holographic complexity and noncommutative gauge theory, J. High Energy Phys. 03 (2018) 108.

[29] B. Swingle and Y. Wang, Holographic complexity of Einstein-Maxwell-Dilaton gravity, J. High Energy Phys. 09 (2018) 106.

[30] M. Moosa, Evolution of complexity following a global quench, J. High Energy Phys. 03 (2018) 031.

[31] B. Chen, W. M. Li, R. Q. Yang, C. Y. Zhang, and S. J. Zhang, Holographic subregion complexity under a thermal quench, J. High Energy Phys. 07 (2018) 034.

[32] H. S. Liu and H. Lu, Action growth of dyonic black holes and electromagnetic duality, J. High Energy Phys. 09 (2019) 102.

[33] A. Bhattacharyya, A. Shekar, and A. Sinha, Circuit complexity in interacting QFTs and RG flows, J. High Energy Phys. 10 (2018) 140.

[34] T. Ali, A. Bhattacharyya, S. Shajidul Haque, E. H. Kim, and N. Moynihan, Time evolution of complexity: A critique of three methods, J. High Energy Phys. 04 (2019) 087.

[35] T. Ali, A. Bhattacharyya, S. Shajidul Haque, E. H. Kim, and N. Moynihan, Post-quench evolution of distance and uncertainty in a topological system: Complexity, entanglement and revivals, arXiv:1811.05985.

[36] S. A. Hosseini Mansoori, V. Jahnke, M. M. Qaemmaqami, and Y.D. Olivas, Holographic complexity of anisotropic black branes, Phys. Rev. D 100, 046014 (2019).

[37] K. Hashimoto, N. Iizuka, and S. Sugishita, Time evolution of complexity in Abelian gauge theories-And playing quantum Othello game -, Phys. Rev. D 96, 126001 (2017).

[38] R. A. Jefferson and R.C. Myers, Circuit complexity in quantum field theory, J. High Energy Phys. 10 (2017) 107.

[39] S. Chapman, M. P. Heller, H. Marrochio, and F. Pastawski, Towards Complexity for Quantum Field Theory States, Phys. Rev. Lett. 120, 121602 (2018).
[40] R.-Q. Yang, A complexity for quantum field theory states and application in thermofield double states, Phys. Rev. D 97, 066004 (2018).

[41] R. Q. Yang, C. Niu, C. Y. Zhang, and K.-Y. Kim, Comparison of holographic and field theoretic complexities for time dependent thermofield double states, J. High Energy Phys. 02 (2018) 082.

[42] R. Q. Yang, Y. S. An, C. Niu, C. Y. Zhang, and K. Y. Kim, More on complexity of operators in quantum field theory, J. High Energy Phys. 03 (2019) 161.

[43] A. R. Brown and L. Susskind, Second law of quantum complexity, Phys. Rev. D 97, 086015 (2018).

[44] A. P. Reynolds and S. F. Ross, Complexity of the AdS soliton, Classical Quantum Gravity 35, 095006 (2018).

[45] P. Caputa, N. Kundu, M. Miyaji, T. Takayanagi, and K. Watanabe, Liouville Action as path-integral complexity: From continuous tensor networks to AdS/CFT, J. High Energy Phys. 11 (2017) 097.

[46] R. Khan, C. Krishnan, and S. Sharma, Circuit complexity in Fermionic field theory, Phys. Rev. D 98, 126001 (2018).

[47] J. Jiang and X. W. Li, Adjusted complexity equals action conjecture, Phys. Rev. D 100, 066026 (2019).

[48] M. Guo, J. Hernandez, R. C. Myers, and S. M. Ruan, Circuit complexity for coherent states, J. High Energy Phys. 10 (2018) 011.

[49] J. Jiang, J. Shan, and J. Yang, Circuit complexity for free Fermion with a mass quench, Nucl. Phys. B954, 114988 (2020).

[50] R. Q. Yang, Y. S. An, C. Niu, C. Y. Zhang, and K. Y. Kim, Principles and symmetries of complexity in quantum field theory, Eur. Phys. J. C 79, 109 (2019).

[51] K. Goto, H. Marrochio, R. C. Myers, L. Queimada, and B. Yoshida, Holographic complexity equals which action?, J. High Energy Phys. 02 (2019) 160.

[52] S. Chapman, J. Eisert, L. Hackl, M. P. Heller, R. Jefferson, H. Marrochio, and R. C. Myers, Complexity and entanglement for thermofield double states, SciPost Phys. 6, 034 (2019).

[53] J. Jiang and X. Liu, Circuit complexity for Fermionic thermofield double states, Phys. Rev. D 99, 026011 (2019).

[54] L. Hackl and R. C. Myers, Circuit complexity for free fermions, J. High Energy Phys. 07 (2018) 139.

[55] R. Nally, Stringy Effects and the role of the singularity in holographic complexity, J. High Energy Phys. 09 (2019) 094.

[56] J. Jiang and H. Zhang, Surface term, corner term, and action growth in $F\left(R_{a b c d}\right)$ gravity theory, Phys. Rev. D 99, 086005 (2019).

[57] J. Jiang and B. X. Ge, Investigating two counting methods of the holographic complexity, Phys. Rev. D 99, 126006 (2019).

[58] S. Chapman, H. Marrochio, and R. C. Myers, Holographic complexity in Vaidya spacetimes. Part I, J. High Energy Phys. 06 (2018) 046.

[59] D. A. Roberts, D. Stanford, and L. Susskind, Localized shocks, J. High Energy Phys. 03 (2015) 051.

[60] S. Chapman, H. Marrochio, and R. C. Myers, Holographic complexity in Vaidya spacetimes. Part II, J. High Energy Phys. 06 (2018) 114. 
[61] J. Jiang, Holographic complexity in charged Vaidya black hole, Eur. Phys. J. C 79, 130 (2019).

[62] L. Susskind and Y. Zhao, Switchbacks and the bridge to nowhere, arXiv:1408.2823.

[63] Z. Y. Fan and M. Guo, Holographic complexity under a global quantum quench, Nucl. Phys. B950, 114818 (2020).

[64] S. Andrews, R. A. Hennigar, and H. K. Kunduri, Chemistry and complexity for solitons in $\mathrm{AdS}_{5}$, arXiv:1912.07637.

[65] A. A. Balushi, R. A. Hennigar, H. K. Kunduri, and R. B. Mann, Holographic complexity and thermodynamic volume, arXiv:2008.09138.

[66] S. S. Hashemi, G. Jafari, and A. Naseh, On the first law of holographic complexity, arXiv:1912.10436.

[67] M. Doroudiani, A. Naseh, and R. Pirmoradian, Complexity for charged thermofield double states, J. High Energy Phys. 01 (2020) 120.

[68] M. Alishahiha, A. Faraji Astaneh, A. Naseh, and M. H. Vahidinia, On complexity for $F(R)$ and critical gravity, J. High Energy Phys. 05 (2017) 009.

[69] S. Lloyd, Ultimate physical limits to computation, Nature (London) 406, 1047 (2000).
[70] K. Landsteiner, Notes on anomaly induced transport, Acta Phys. Pol. B 47, 2617 (2016).

[71] J. B. Gutowski and H. S. Reall, Supersymmetric AdS5 black holes, J. High Energy Phys. 02 (2004) 006.

[72] M. Cvetic, H. Lu, and C. N. Pope, Charged Kerr-de Sitter black holes in five dimensions, Phys. Lett. B 598, 273 (2004).

[73] M. Cvetic, H. Lu, and C. N. Pope, Charged rotating black holes in five dimensional $\mathrm{U}(1)^{3}$ gauged $\mathcal{N}=2$ supergravity, Phys. Rev. D 70, 081502 (2004).

[74] Z.-W. Chong, M. Cvetic, H. Lu, and C. N. Pope, General Nonextremal Rotating Black Holes in Minimal FiveDimensional Gauged Supergravity, Phys. Rev. Lett. 95, 161301 (2005).

[75] A. Al Balushi and R. B. Mann, Null hypersurfaces in Kerr(A)dS spacetimes, Classical Quantum Gravity 36, 245017 (2019).

[76] M. T. N. Imseis, A. A. Balushi, and R. B. Mann, Null hypersurfaces in Kerr-Newman-AdS black hole and super-entropic black hole spacetimes, arXiv:2007.04354. 